\title{
Outcomes of a novel intrathoracic esophagogastric anastomotic technique
}

\author{
Kenneth A. Kesler, MD, ${ }^{\mathrm{a}}$ Neal K. Ramchandani, MD, ${ }^{\mathrm{a}}$ Shadia I. Jalal, MD, ${ }^{\mathrm{b}}$ Samatha M. Stokes, MS, \\ Mark R. Mankins, MD, ${ }^{a}$ DuyKhanh Ceppa, MD, ${ }^{a}$ Thomas J. Birdas, MD, ${ }^{a}$ Panos N. Vardas, MD, ${ }^{a}$ and \\ Karen M. Rieger, MD
}

\begin{abstract}
Objectives: Anastomotic complications represent a significant source of morbidity and occasionally mortality after esophagectomy. Since 2009, we have used a novel "side-to-side: staple line-on-staple line" (STS) technique for intrathoracic esophagogastric anastomoses, designed to create a wide-diameter esophagogastric anastomosis while preserving stomach conduit blood supply. In this study, we describe the technique and review outcomes of our institution's initial 6-year experience.
\end{abstract}

Methods: An institutional database query identified 278 consecutive patients who underwent Ivor Lewis esophagogastrectomy using an STS esophagogastric anastomotic technique from 2009 through 2015 . A retrospective review was conducted to assess outcomes with a focus on anastomotic complications.

Results: There were a total of $8(2.9 \%)$ anastomotic leaks in patients who underwent STS esophagogastric anastomosis, 3 of which were grade I/II leaks and required no intervention. There was a leak rate of 6.3\% ( 2 of 32) after esophagectomy for benign conditions (both leaks occurring in 8 total patients $(25 \%)$ who received surgery for end-stage achalasia) compared with a $2.4 \%$ leak rate (6 of $246)$ in whom esophagectomy was performed for malignancy $(P=.22)$. Fourteen patients $(5.0 \%)$ required a median of 2 dilatations for anastomotic stricture after STS anastomosis. Supplemental jejunostomy feedings were required in only $11.1 \%$ of these patients after hospital discharge.

Conclusions: We believe this novel STS technique provides excellent results with respect to the incidence of intrathoracic esophagogastric anastomotic leak and stricture after esophagectomy. Additionally this technique has significantly reduced the need for enteral feeding after hospital discharge. (J Thorac Cardiovasc Surg 2018;156:1739-45)

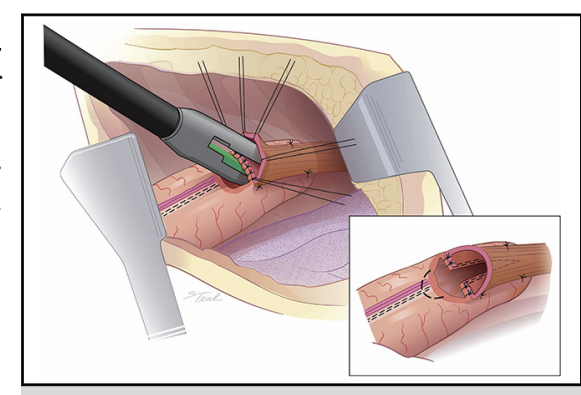

An endostapler is used to cut through and restaple the lesser curve conduit staple line.

\section{Central Message}

In this report, a novel intrathoracic esophagogastric anastomotic technique designed to create a wide-diameter anastomosis while preserving conduit blood supply is described. Outcomes are reviewed.

\section{Perspective}

Anastomotic complications represent a significant source of morbidity and occasionally mortality after esophagectomy. We describe a novel technique for intrathoracic esophagogastric anastomoses, designed to create a widediameter esophagogastric anastomosis while preserving stomach conduit blood supply. We report outcomes of the first 278 consecutive patients who underwent this technique.

See Editorial Commentary page 1746
Esophagogastric anastomotic leak represents a significant source of perioperative morbidity and occasionally mortality after esophagectomy. ${ }^{1}$ Additionally complications including anastomotic leak, have been directly linked to many adverse

\footnotetext{
From the ${ }^{\mathrm{a}}$ Division of Cardiothoracic, Department of Surgery, and ${ }^{\mathrm{b}}$ Medical Oncology Division, Department of Medicine, Indiana University Melvin and Bren Simon Cancer Center, Indianapolis, Ind.

Received for publication Jan 11, 2018; revisions received May 4, 2018; accepted for publication May 9, 2018; available ahead of print July 19, 2018.

Address for reprints: Kenneth A. Kesler, MD, Thoracic Surgery Division, Department of Surgery, Indiana University, Barnhill Drive EM \#212, Indianapolis, IN 46202 (E-mail: kkesler@iupui.edu).

$0022-5223 / \$ 36.00$

Copyright (c) 2018 by The American Association for Thoracic Surgery

https://doi.org/10.1016/j.jtcvs.2018.05.088
}

long-term outcomes including hospital readmission, poor quality of life, and even early mortality. ${ }^{2-4}$ Anastomotic stricture also represents a common complication after esophagectomy, which is closely associated with anastomotic leak. ${ }^{5}$ Anastomotic strictures not only negatively affect quality of life but also increase cost of care when dilatation is required.

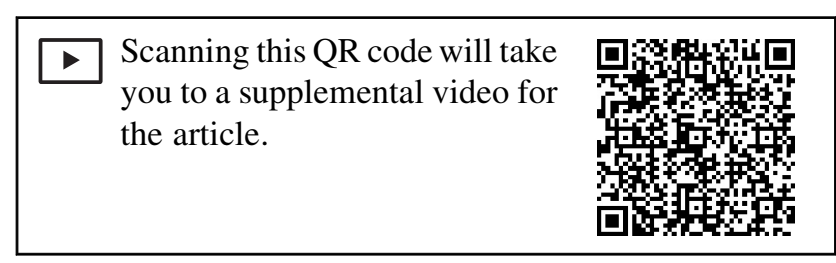




\section{Abbreviations and Acronyms}

STS $=$ side-to-side: staple line-on-staple line $\mathrm{EEA}=$ end-to-end anastomotic stapler

A query of the Society of Thoracic Surgeons General Thoracic Database reported a leak rate of $9.3 \%$ in patients who underwent intrathoracic esophagogastric anastomoses. ${ }^{6}$ Although the mortality rate of anastomotic leak appears to be decreasing, subsequent stricture rates remain relatively high ranging between 10\% and 56\%. In 1998 and 2000, Collard and colleagues ${ }^{8}$ and Orringer and colleagues ${ }^{9}$ proposed the utility of a linear stapler to establish a side-to-side cervical esophagogastric anastomosis. The posterior triangulated opening created with the linear stapler was shown to be not only less prone to leak but also less predisposed to stricture formation.

Since 2009, we have used a novel "side-to-side: staple line-on-staple line" (STS) technique for intrathoracic esophagogastric anastomoses, designed to create a wide diameter esophagogastric anastomosis while preserving stomach conduit blood supply aimed at reducing anastomotic leaks and strictures. In this report, we describe this esophagogastric anastomotic technique and review outcomes after the first 6 years of our experience.

\section{METHODS}

Under an approved institutional review board protocol, an institutional database was queried to identify consecutive patients who underwent Ivor Lewis esophagogastrectomy using an STS esophagogastric anastomotic technique from 2009 through 2015 (Video 1). Demographic data including patient age, sex, diagnosis, neoadjuvant chemotherapy/radiation therapy in cases of malignancy, and comorbidities were collected. All patients underwent radiologic evaluation of the esophagogastric anastomosis usually with water-soluble contrast under fluoroscopy or occasionally computed tomography imaging typically from 5 to 7 days postoperatively. Postoperative outcomes, including anastomotic leak, grade of leak, and anastomotic stricture requiring dilatation, were recorded..$^{10}$

\section{Description of the STS Technique}

An upper midline laparotomy is used. A wide Kocher maneuver is performed, which not only tends to straighten the gastric outlet to optimize emptying but also allows well vascularized portions of the stomach conduit to be advanced to or above the azygous arch for anastomosis. A laparotomy sponge is temporarily placed under the duodenum and head of pancreas to elevate the conduit. The sponge is removed after return to the abdomen during the third phase of the procedure. A nasogastric tube is positioned with the tip just proximal to the pylorus and used as a "handle" retracting the stomach cephalad while dividing the gastrocolic ligament, avoiding manipulation of the right gastroepiploic vessels.

After the stomach, intra-abdominal esophagus, and upper abdominal lymph nodes in cases of malignancy, are mobilized, the lesser curve is cleared of fat and blood vessels typically 3 to $5 \mathrm{~cm}$ distal to the gastroesophageal junction. Stomach geometries vary as do locations of tumors. Optimal surgical plans are to achieve a 3- to 5-cm distal stomach and 5- to 7-cm proximal esophageal margin if possible in cases of malignancy. For cardia tumors, the lesser curve is usually cleared closer to the incisura for an adequate distal stomach margin, however, results in a shorter conduit anticipating esophagogastric anastomosis near the level

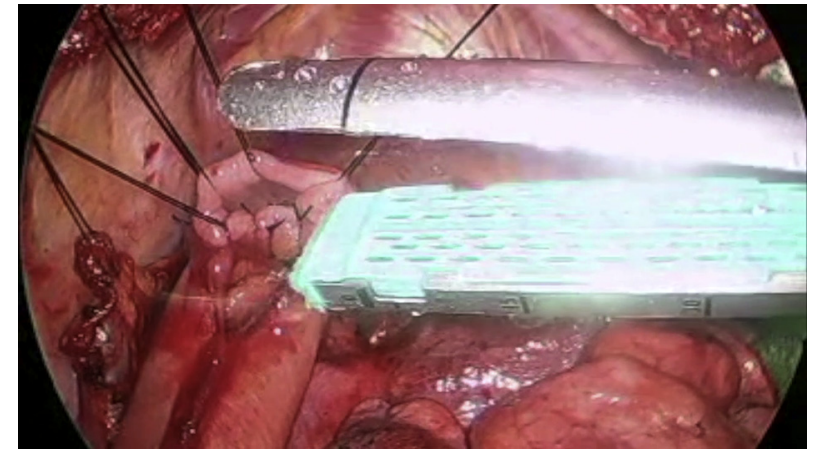

VIDEO 1. Side-to-side: staple line-on-staple line technique for intrathoracic esophagogastric anastomosis. The critical steps of this technique are presented. Video available at: https://www.jtcvs.org/article/ S0022-5223(18)31524-1/fulltext.

of the tracheal carina. Conversely for tumors approaching the mid esophagus level requiring a high anastomosis near the intrathoracic inlet, the lesser curve is cleared closer to the gastroesophageal junction to preserve conduit length. The upper fundus and cardia are cleaved from the rest of the stomach with 3 to 4 fires of a $60-\mathrm{mm}$ endoscopic stapler (Ethicon Endo-Surgery, Echelon Flex 60, "black" tristapler cartridge; Ethicon Corp, Bridgewater, NJ) into the cleared lesser curve area, which allows the conduit to be delivered out of the abdomen. Lesser omental fat around the right gastric vessels can tether the lesser curve. Carefully debulking of this tissue will straighten and lengthen the conduit.

The stomach is secured at 3 points, which are retracted outward. A relatively narrow but not too narrow uniform diameter conduit is created with an initial fire of a 100-mm stapler (Covidien [Dublin, Ireland], ILA Autosuture, "green" cartridge, 2 rows of $4.8 \mathrm{~mm}$ staples) aiming just beneath the previous staple line on the lesser curve (Figure 1). Completion of conduit construction usually requires 1 or 2 additional fires of a $60-\mathrm{mm}$ endoscopic stapler (Ethicon Endo-Surgery, Echelon Flex 60, "black" tristapler cartridge). Aiming the stapler just beneath the previous staple line on the lesser curve typically creates a straight conduit with a diameter averaging 5 to $7 \mathrm{~cm}$ while preserving right gastric vessel contribution to the conduit. The crescent-shaped stomach defect is sent to pathology for frozen section analysis when surgery is being done for malignancy. The tip of the conduit is inspected and punctate bright red blood is usually seen oozing through the lesser curve staple line within 5 to 10 minutes signifying good conduit perfusion. If no punctate bright red blood oozing is present, the conduit tip is slightly trimmed back with another 100-mm stapler using standard green cartridge until punctate bright red bleeding is seen. Tristaplers are not used for conduit tip trimming because cutting through and restapling the conduit tip to establish the anastomosis in the chest might be difficult. A pyloric opening procedure is performed to assure that all conduits empty as well as possible, typically using a standard Heineke-Mikulicz pyloroplasty. The pylorus is opened longitudinally until a finger can be admitted into the stomach antrum then closed transversely in 2 layers of sutures. After the pyloroplasty is complete, the conduit is stretched cephalad to get an estimation of how high the conduit will reach in the chest. In rare cases, if it is determined that length of the conduit might be too foreshortened by a pyloroplasty, circumferential injection of botulism toxin in the pyloric muscle can alternatively be used. The right pleura is opened widely through the diaphragm crus then the tip of the conduit is placed into the right chest keeping the conduit oriented with the lesser curve staple line facing rightward. The abdomen is temporarily closed with a few interrupted facial sutures and skin staples.

The patient is placed in the left lateral decubitus position, and right thoracotomy, performed typically through the fifth interspace, sparing the serratus anterior muscle. The intercostal muscle is divided underneath the incision to within 3 to $5 \mathrm{~cm}$ of the vertebral body posteriorly and 


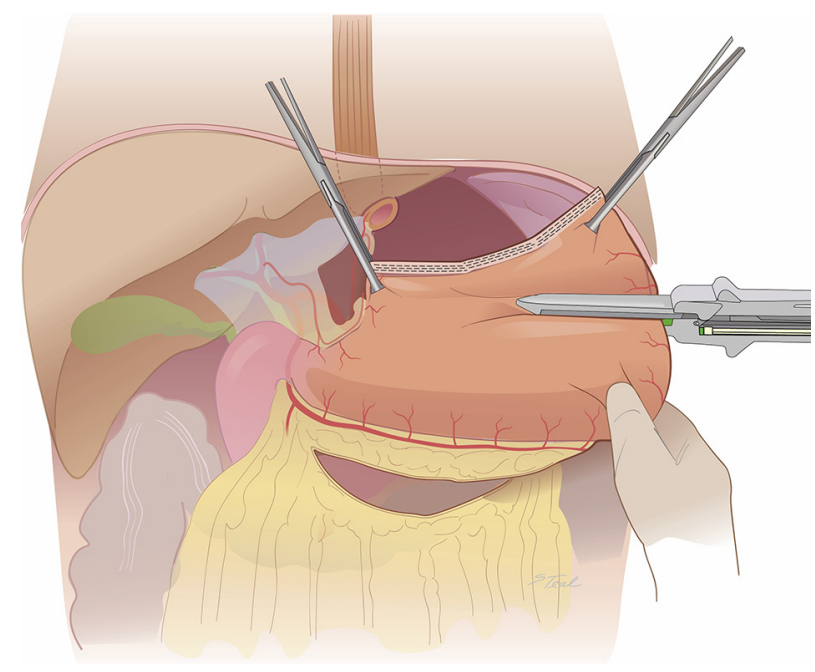

FIGURE 1. The stomach is secured at 3 points, which are retracted outward. A relatively uniform diameter conduit is created with staplers aimed just inferior to the previous staple line on the lesser curve.

sternum anteriorly which "unhinges" the rib. This maneuver, along with slow progressive rib spreading over the course of the thoracic phase, minimizes the risk of rib fracture/rib bruising and in our experience most patients can rapidly taper postoperative narcotics after chest tube removal. The arch of the azygous vein is excised. The inferior pulmonary ligament is divided. For malignancy, en bloc dissection is continued, mobilizing the distal two-thirds of the intrathoracic esophagus and surrounding soft tissues from pericardium anteriorly to aorta posteriorly. The conduit is pulled into the chest until there is no redundancy. The conduit is digitally examined at the crus to make sure the right gastric and right gastroepiploic vascular pedicles are not being tethered preventing full delivery of the conduit. At the level of the carina, the esophagus itself is dissected from surrounding mediastinal soft tissues just to the point where the tip of the conduit comfortably reaches to perform the anastomosis without tension and avoid unnecessary esophageal devascularization, which is usually 3 to $5 \mathrm{~cm}$ superior to the carina. Anastomoses can, however, be established nearer the carina for cardia tumors and conversely at the thoracic inlet for tumors involving the middle intrathoracic esophagus.

The mid left lateral aspect of the upper third of the intrathoracic esophagus is aligned over the lesser curve staple line with 4 initial tacking sutures approximately 2 to $3 \mathrm{~cm}$ apart (Figure 2). A shorter side-to-side anastomosis can be performed if needed to achieve an adequate proximal esophageal margin. The esophagus is transected $1 \mathrm{~cm}$ distal to the inferior set of tacking sutures and in cases of malignancy a ring of esophagus from the surgical specimen sent for frozen section pathologic examination before proceeding. A corresponding $1-$ to $2-\mathrm{cm}$ opening is made in the conduit across the lesser curve staple line. More tacking sutures are placed between the adjacent conduit and esophageal walls beginning in the middle through the lesser curve staple line then 2 sutures on either side (Figure 3). The narrow anvil of an endoscopic stapler (Ethicon Endo-Surgery, EZ 45, "green" cartridge, 4.1-mm staples) with the small anvil placed in the esophagus and the large anvil in the conduit (see Appendix 1). The stapler is fired cutting through and restapling the lesser curve staple line (Figure 4). The side-to-side communication is inspected to assure complete cutting and stapling has occurred. The length of the cut end of the esophagus is typically somewhat longer than the edge of the gastric conduit, so an ellipse of conduit is removed over the lesser curve staple line rather than extending the rent laterally to preserve conduit collateral blood supply (Figure 4, inset). The open common lumen is closed in 2 layers of sutures beginning with an inner layer of inverted interrupted 3-0 polyglactin suture followed by a second layer of interrupted 3-0 silk suture using a Lembert technique

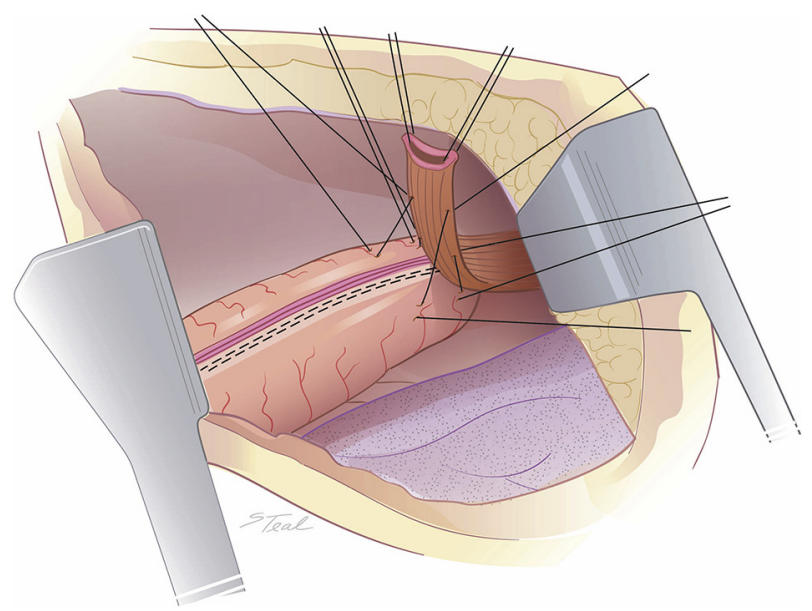

FIGURE 2. The mid left lateral aspect of the upper third of the intrathoracic esophagus is aligned over the lesser curve staple line at the tip of the conduit with 4 initial tacking sutures approximately 2 to $3 \mathrm{~cm}$ apart.

(Figure 5). Suture closure of the open common lumen usually eliminates any residual conduit redundancy although the conduit length should be gaged so there is no tension on this suture line. A pleural flap mobilized from over the upper third of the intrathoracic esophagus is then tacked to the conduit with interrupted sutures (Figure 6). More recently we have favored using a pericardial fat pad mobilized from the anterior mediastinum or as a second option pleura mobilized from the posterior body wall to loosely wrap around the anastomosis. The superior aspect of the lesser curve conduit staple line is oversewn using a Lembert technique inferiorly until the right gastric vessels are encountered. A nasogastric tube passed into the conduit to level of crus and two 28-French chest tubes placed, one anterior and the other posterior, next to but not abutting the conduit, before chest closure. Chest tubes are placed to underwater seal only.

The third phase of the procedure involves returning the patient to the supine position and reopening the laparotomy ("double flip"). Any tethered right gastroepiploic fat is gently pushed up into the chest then the right gastroepiploic fat and conduit carefully tacked to the crus with interrupted 2-0 silk sutures (Figure 7). A feeding jejunostomy tube is

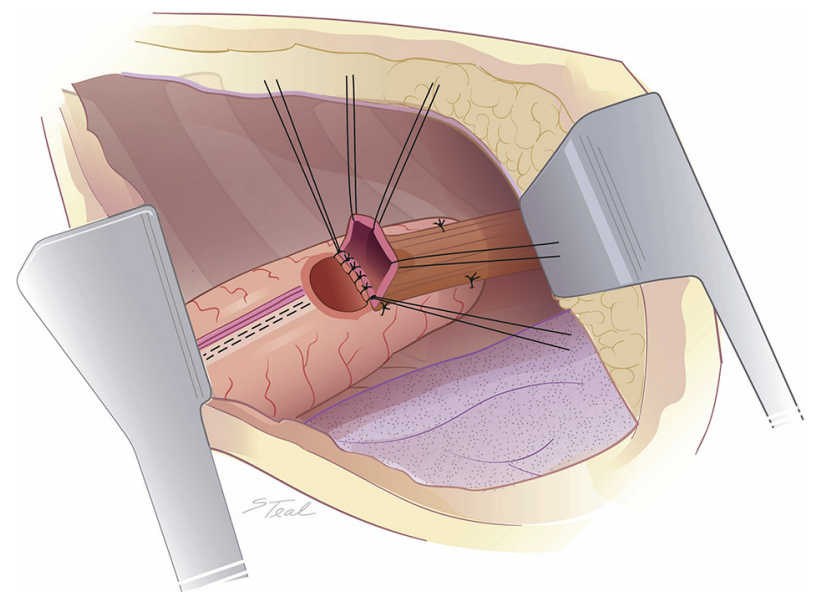

FIGURE 3. A 1- to $2-\mathrm{cm}$ rent is made in the stomach conduit across the lesser curve staple line. Tacking sutures are placed between the adjacent conduit and esophageal walls beginning in the middle through the lesser curve staple line then 2 sutures on either side. 


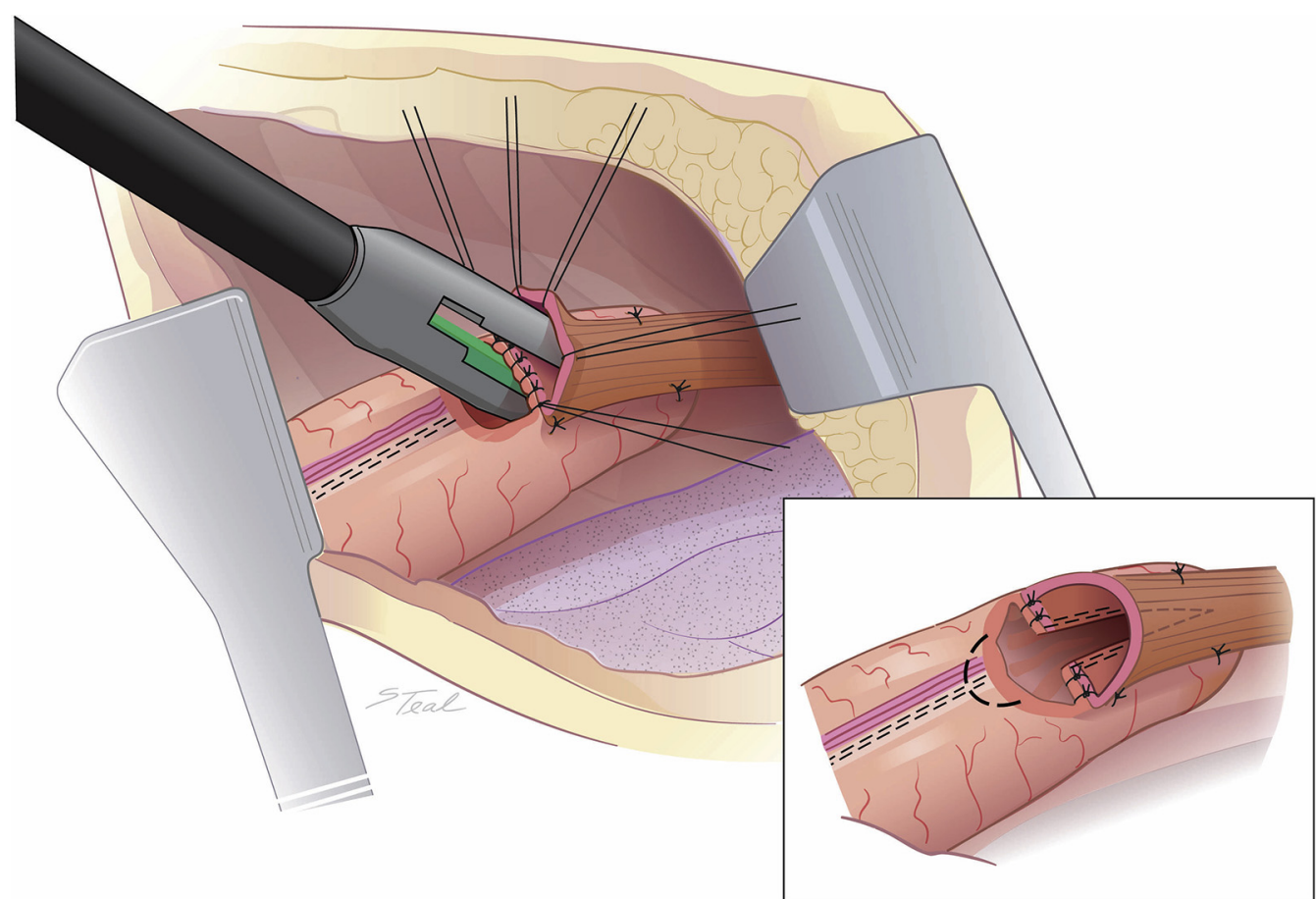

FIGURE 4. A 45-mm endoscopic stapler is fired cutting through and restapling the lesser curve staple line. Typically only two-thirds of the stapler length is used. An ellipse of conduit is removed over the lesser curve staple line (dotted line) rather than extending the rent laterally to equalize the length of the cut end of the esophagus and gastrotomy (inset).

placed in select patients, primarily elderly patients or patients with preoperative malnutrition, then the laparotomy incision closed. Patients remain intubated the evening of surgery with extubation planned the next morning. Pain control is provided by epidural catheters placed before anesthetic induction for the first 3 to 4 days supplemented by intravenous narcotics. Historically, a nasogastric tube was maintained for 7 postoperative days at which time a contrast upper gastrointestinal study was obtained. More recently, contrast upper gastrointestinal studies have been obtained 5 to 6 days postoperatively to facilitate earlier discharge. If no anastomotic leak is identified, then the nasogastric tube is removed and the patient advanced to a soft diet over a 3-day interval before discharge. Patients are instructed to advance to a regular diet after 2 weeks as tolerated.

\section{RESULTS}

A total of 278 patients were identified who underwent an STS intrathoracic esophagogastric anastomosis. Patient demographic characteristic are given in Table 1. In summary, most patients $(82.0 \%)$ were male at an average age of 60 years (range, 23-84 years). Surgery was performed for neoplasms and benign etiologies in 246 and 32 patients, respectively, with $71.9 \%$ of patients receiving chemotherapy and radiation therapy before surgery.

Overall, 115 patients $(41.4 \%)$ experienced postoperative morbidity including 64 patients with pulmonary complications (including reintubation/therapeutic bronchoscopy, $\mathrm{n}=22$; pneumonia, $\mathrm{n}=17$; and prolonged chest tube drainage $>10$ days/thoracentesis, $\mathrm{n}=19$ ), 58 and 41 patients experiencing cardiac (atrial tachyarrhythmias, $\mathrm{n}=55$ ), and other complications (including wound infection, $\mathrm{n}=7$; transient renal insufficiency, $\mathrm{n}=6$; chylothorax, $\mathrm{n}=5$; and ileus, $\mathrm{n}=5$ ). During the first half of the series, the median hospital stay was 13 days, ranging from 9 to 86 days. Over the second half of this series, the median hospital stay was reduced to 11 days. There were 3 surgical deaths $(1.1 \%), 2$ secondary to respiratory complications and 1 because of cardiac failure. None of these 3 patients had an anastomotic complication.

With respect to anastomotic complications, overall 8 patients $(2.9 \%)$ had an anastomotic leak (Table 2). Three of these patients had a grade I/II leak, which resolved without intervention. Five patients with grade III leaks were treated with endoscopic stent placement and pleural drainage. No patient sustained a grade IV leak. Patients who underwent esophagectomy for benign conditions had leak rate of $6.3 \%$ compared with a $2.4 \%$ leak rate in patients who underwent surgery for malignancy $(P=.22)$. In particular, 2 of 8 patients $(25.0 \%)$ who underwent intrathoracic STS anastomosis for end-stage achalasia experienced a leak. Fourteen patients $(5.0 \%)$ required postoperative dilatation of an anastomotic stricture, one of whom showed a leak on postoperative contrast study. Five patients required 1 dilatation, 6 patients required between 2 and 4 dilatations, and 3 patients required 5 or more dilatations. Overall, 118 patients $(42.4 \%)$ had jejunostomy tubes placed at the time of surgery, however, only 28 patients $(10.0 \%)$ required 


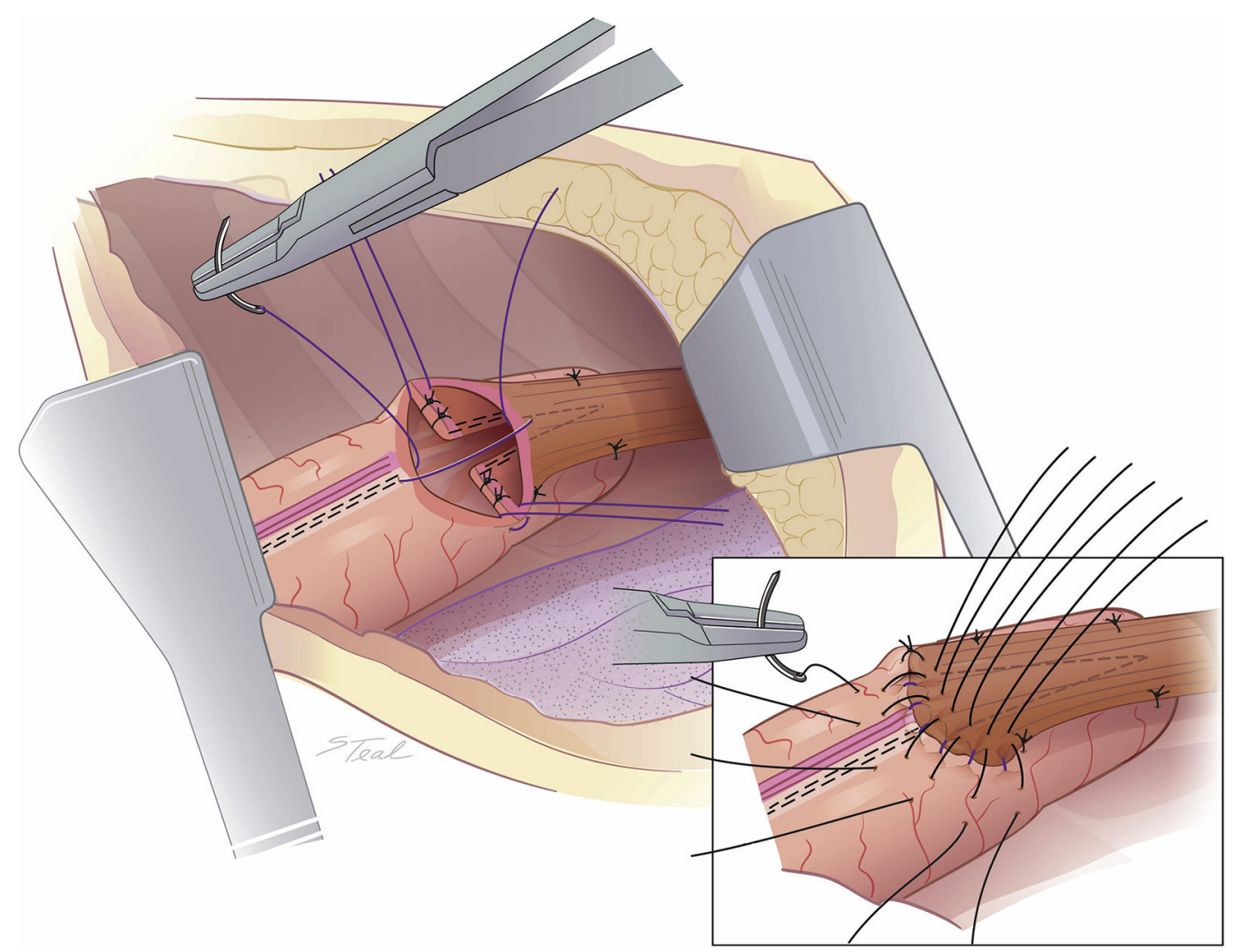

FIGURE 5. The open common lumen is manually closed in 2 layers of sutures beginning with inverted interrupted absorbable sutures. The first suture layer is imbricated by a second layer of Lembert silk sutures, placing the stomach suture a few millimeters inferior to the first layer of sutures (inset).

jejunostomy tube feedings after discharge. Three patients who did not have a jejunostomy tube placed at time of surgery had jejunostomy tubes placed because of poor oral intake within the first 3 months after surgery equating to an $11.1 \%$ rate of jejunostomy tube usage. Patients were followed for a range of 1 to 61 months. Of the 246 patients who underwent surgery for malignancy, at last follow-up 138 were alive without disease, 33 were alive with disease, and 75 had died. Twenty-eight of 32 patients (87.5\%) who underwent esophagectomy for benign disease are alive and well. Two patients $(0.7 \%)$ developed a diaphragmatic hernia requiring repair.

\section{DISCUSSION}

Esophagectomy is a large complex procedure usually performed for malignancy and like any other large complex surgery performed for cancer, precise execution of several sequential steps is critical for uneventful healing as well as a good oncologic outcome. Restoring gastrointestinal function is additionally necessary during esophagectomy and also requires performance of several critical steps. As a significant number of esophageal cancer patients are living longer as a result of early detection and multimodality therapy, and optimizing upper gastrointestinal tract function also becomes increasingly important. Gastric conduit construction including diameter, length, and orientation can all affect upper gastrointestinal tract function. Ingested food and liquid have the potential to hang up in 3 locations, the esophagogastric anastomosis, stomach body, and gastric outlet. Poor conduit "function," the term for which is a bit of an oxymoron in the case of a passive stomach conduit, is not infrequently a result of technical issues in any or all of these 3 locations. Anastomotic strictures and poor conduit function for any reason might significantly negatively affect disease-specific quality of life and additionally increases costs when dilatation is required. ${ }^{2,5}$

Linear stapled techniques as described by Collard and colleagues $^{8}$ and Orringer and colleagues ${ }^{9}$ for cervical esophagogastric anastomoses would seem to have similar advantages of anastomoses less prone to leak and stricture when performed in an intrathoracic position. An experience from Mayo Clinic showed a 5.6\% incidence of intrathoracic anastomotic leak in 177 patients where linear stapled anastomosis was performed compared with an $8.3 \%$ leak rate in 48 patients who underwent anastomosis with an end-to-end anastomotic stapler (EEA). ${ }^{5}$ In this study, strictures occurred in nearly half of patients with anastomotic leak and leak was significantly predictive of stricture. Moreover, stricture formation in the subset of patients undergoing intrathoracic anastomoses were significantly reduced 


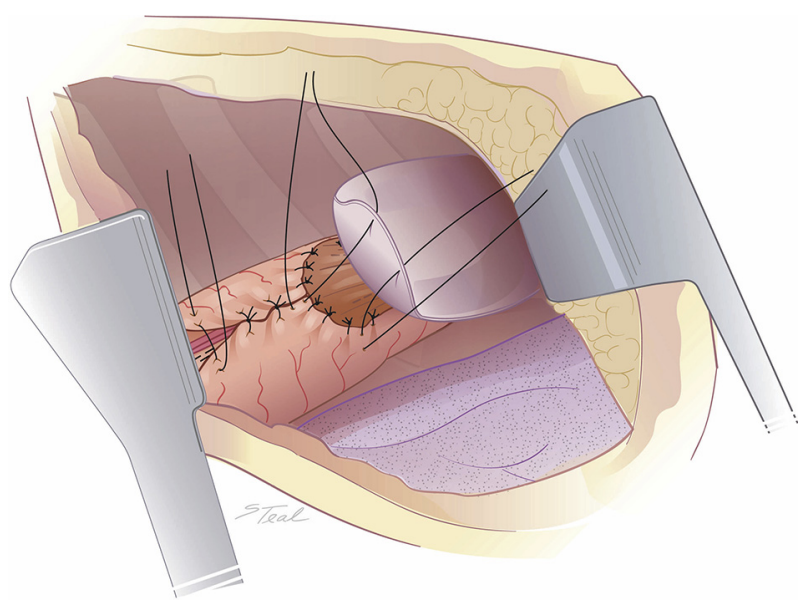

FIGURE 6. The superior aspect of the lesser curve conduit staple line can be oversewn at this point with interrupted silk sutures inferiorly until the right gastric vessels are encountered. The anastomosis is covered by a flap of pleura mobilized from the upper chest or lateral body wall. More recently, we have also used a loose wrap of pericardial fat or internal mammary artery vascularized pedicle flaps.

with a linear stapler compared with EEA or manually sewn techniques. The 1-year probability of stricture for EEA anastomoses was $32 \%$ compared with $8.6 \%$ with a linear stapled techniques. Other studies have shown the superiority of linear-stapled over EEA anastomoses with respect to reducing stricture formation. Wang and colleagues randomized 155 patients to 3 esophagogastric anastomotic

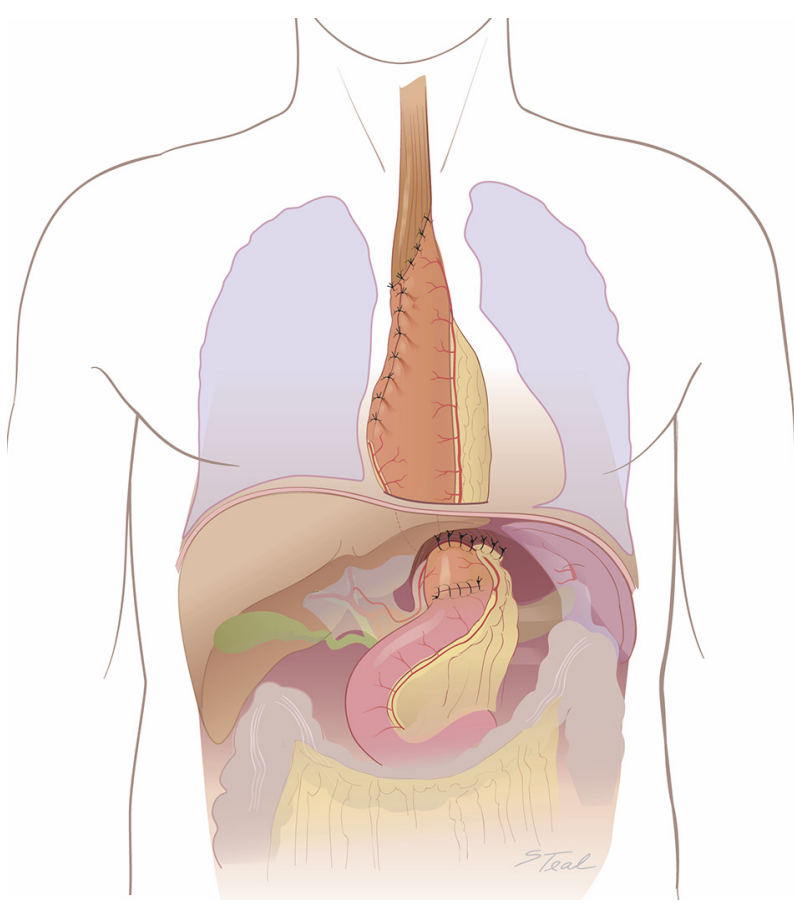

FIGURE 7. The laparotomy incision is reopened. The right gastroepiploic fat and conduit carefully tacked to the crus with 2-0 silk sutures.
TABLE 1. Patient demographic characteristics

\begin{tabular}{lc}
\hline \multicolumn{1}{c}{ Characteristic } & Value \\
\hline Age & $60.3 \pm 11.4(23-84)$ \\
Sex & $228(82.0)$ \\
Male & $50(18.0)$ \\
Female & \\
Comorbidity & $114(41.0)$ \\
Cardiac & $70(25.2)$ \\
Diabetes & $32(11.5)$ \\
COPD & $200(71.9)$ \\
Neoadjuvant Tx & \\
Diagnosis & \\
Malignancy & \\
$\quad$ Adenocarcinoma & $237(85.3)$ \\
Other* & $9(3.2)$ \\
Benign $\dagger$ & $32(11.5)$ \\
\hline
\end{tabular}

Age is given as mean years \pm SD (range). Discrete variables are given as patient numbers with percentage of series in parentheses. COPD, Chronic obstructive pulmonary disease; Neoadjuvant $T x$, chemotherapy and radiation therapy. *Includes squamous-cell cancer, neuroendocrine neoplasms, and germ cell tumors. †ंIncludes achalasia, stricture, Barrett esophagus, leiomyoma, and esophageal perforation.

techniques. ${ }^{11}$ Stricture rates were $0 \%$ in patients who underwent a linear stapled technique compared with $9.6 \%$ and $19.1 \%$ in the manually sewn and circular stapled cases, respectively; the differences of which were statistically significant. Our overall $2.9 \%$ and $5.0 \%$ rate of anastomotic leak and stricture, respectively, using this STS technique compare very favorably with previous publications including the intrathoracic leak rate of $9.3 \%$ reported from the Society of Thoracic Surgeons database. ${ }^{6}$

Common to all previous reports, linear stapled anastomoses performed in the chest are placed on the anterior stomach conduit wall, which has potential to create an ischemic strip of conduit between the lesser curve staple line and anastomosis. Novel to our technique, collateral blood supply to the conduit tip is preserved by cutting through and restapling the lesser curve staple line. Mechanical staplers are designed to reliably cut through and restaple previous staple lines. In our experience, however, cutting through a staple line established with a tristapler is difficult and we therefore believe that tristaplers should be avoided at the tip of the

TABLE 2. Anastomotic leak

\begin{tabular}{lc}
\hline & Value \\
\hline Total & $8(2.9)$ \\
Malignant & $6(2.4)$ \\
Benign & $2(6.3)$ \\
Grade & \\
I & 2 \\
II & 1 \\
III & 5 \\
IV & 0 \\
\hline
\end{tabular}

Data are presented as patient numbers, with percentage of series in parentheses. 
conduit with this technique. Although on rare occasions we have experienced minor incomplete tissue division with the stapler blade, staples themselves have uniformly seated well, and any incompletely divided tissue been easily divided under direct vision with a scissors. After an initial concern, we now believe that perfect alignment of the linear stapler over the previous lesser curve staple line, although optimal and usually achieved, is not necessary and close alignment is sufficient. Fundamentally, in addition to preserving collateral vessels to the conduit tip, the conduit itself must have good blood supply. In our experience, the presence of punctate red bleeding at the tip of the lesser curve staple line has been a very reliable indicator of good conduit perfusion. A wide Kocher maneuver also allows advancement of the most vascularized portions of the stomach conduit high into the chest in this regard.

There are some limitations to this study including the retrospective nature and limitations to the technique itself. Of note however, this approach was uniformly used with an "intent to treat" in all patients who underwent surgery for mid esophageal to proximal stomach pathology, including acute perforations in stable patients not amenable to primary repair over the study interval. First, the sensitivity of contrast esophagram to detect an anastomotic leak is not $100 \%$ with occasional failure to show small well contained areas of leak. Upper gastrointestinal contrast studies, however, did show prompt conduit emptying with minimal to no reflux in the upright position, signifying good function in most patients. Next, inherent to all intrathoracic side-to-side anastomotic techniques, is the need to dissect an additional 3 to $4 \mathrm{~cm}$ of residual esophagus, potentially decreasing the length of proximal esophageal margin in cases of malignancy as well as resulting in some degree of esophageal devascularization. For long or more central tumors where an esophagogastric anastomosis needs to be established near the thoracic inlet to achieve an adequate margin, we have used a somewhat shorter initial side-to-side communication. To avoid excessive esophageal devascularization, it is important not to dissect the intrathoracic esophagus any further superior than the tip of the conduit will comfortably reach. We speculate the higher incidence of leak for achalasia using this STS technique might be related to devascularization of a thickened esophageal wall where the blood supply is already compromised. Esophageal dilatation secondary to achalasia also makes the manually sewn open end difficult and might factor into higher leak rates. On the basis of our experience, we have abandoned performing STS intrathoracic anastomoses in these cases. Finally, most leaks in this series were observed at the junction of the lesser curve stomach staple line and esophagus at the mid portion of the open common lumen suture line. This finding underscores the need to eliminate tension and perhaps use bulkier more vascularized soft tissue coverage in this area.

Esophagectomy plays an important role in the treatment of esophageal cancer and select benign conditions. Esophagogastric anastomotic complications are associated with morbidity, occasional mortality, and increased costs of care. We believe this novel STS technique is easily adapted, reproducible, and provides excellent results with respect to minimizing anastomotic complications. Conduit construction/orientation as described might additionally optimize functional outcomes as reflected by our low need for supplemental jejunostomy feedings. Future plans include studying long-term outcomes, including esophageal-specific quality of life assessment.

\section{Conflict of Interest Statement}

Authors have nothing to disclose with regard to commercial support.

\section{References}

1. Cassivi SD. Leaks, strictures, and necrosis: a review of anastomotic complications following esophagectomy. Semin Thorac Cardiovasc Surg. 2004;16: 124-32.

2. Low DE, Alderson D, Cecconello I, Chang AC, Darling GE, D'Journo XB, et al. International consensus on standardization of data collection for complications associated with esophagectomy:esophagectomy complication consensus group (ECCG). Ann Surg. 2015;262:286-94.

3. Derogar M, Orsini N, Sadr-Azodi O, Lagergren P. Influence of major postoperative complications on health-related quality of life among long-term survivors of esophageal cancer surgery. J Clin Oncol. 2012; 30:1615-9.

4. Kofoed SC, Calatayud D, Jensen LS, Helgstrand F, Achiam MP, De Heer P, et al. Intrathoracic anastomotic leakage after gastroesophageal cancer resection is associated with increased risk of recurrence. J Thorac Cardiovasc Surg. 2015; 150:42-8.

5. Price TN, Nichols FC, Harmsen WS, Allen MS, Cassivi SD, Wigle DA, et al. A comprehensive review of anastomotic technique in 432 esophagectomies. Ann Thorac Surg. 2013;95:1154-61.

6. Kassis ES, Kosinski AS, Ross P Jr, Koppes KE, Donahue JM, Daniel VC. Predictors of anastomotic leak after esophagectomy: an analysis of the Society of Thoracic Surgeons general thoracic database. Ann Thorac Surg. 2013;96: 1919-26.

7. Yuan Y, Wang KN, Chen LQ. Esophageal anastomosis. Dis Esophagus. 2015;28: 127-37.

8. Collard JM, Romagnoli R, Goncette L, Otte JB, Kestens PJ. Terminalized semimechanical side-to-side suture technique for cervical esophagogastrostomy. Ann Thorac Surg. 1998;65:814-7.

9. Orringer MB, Marshall B, Iannectoni MD. Eliminated the cervical esophagogastric anastomotic leak with a side-to-side stapled anastomosis. J Thorac Cardiovasc Surg. 2000;119:277-88.

10. Lerut T, Coosemans W, Decker G, De Leyn P, Nafteux P, van Raemdonck D Anastomotic complications after esophagectomy. Dig Surg. 2002;19:92-8.

11. Wang WP, Gao Q, Wang KN, Shi H, Chen LQ. A prospective randomized controlled trial of semi-mechanical versus hand-sewn or circular stapled esophagogastrostomy for prevention of anastomotic stricture. World J Surg. 2013;37: 1043-50.

Key Words: esophagectomy, esophageal cancer, esophagectomy complications 


\section{APPENDIX 1. ADDENDUM}

Since completion of this study, Ethicon Corporation (Bridgewater, NJ) has discontinued production of the EZ 45 endostapler. We have substituted the EZ 45 with the Ethicon Endo-Surgery, Echelon Flex 45, "green" cartridge, 4.1$\mathrm{mm}$ staple height, shaving off the distal $5 \mathrm{~mm}$ of the plastic tip with an oscillating saw to simulate the shorter length of the EZ 45 to optimize of the length of the side-to-side communication between the esophagus and stomach conduit. To date, we have used this stapler substitution in 136 patients who underwent STS intrathoracic esophagogastric anastomosis with outcomes consistent with our experience using the EZ 45. 